\title{
BMJ Open Assessment of the Cow's Milk-related Symptom Score (CoMiSS) as a diagnostic tool for cow's milk protein allergy: a prospective, multicentre study in China (MOSAIC study)
}

Yvan Vandenplas, ${ }^{1}$ Zheng-Yan Zhao, ${ }^{2}$ Rajat Mukherjee, ${ }^{3}$ Christophe Dupont, ${ }^{4}$ Philippe Eigenmann, ${ }^{5}$ Mikael Kuitunen, ${ }^{6}$ Carmen Ribes Koninckx, ${ }^{7}$ Hania Szajewska, ${ }^{8}$ Andrea von Berg, ${ }^{9}$ Kateřina Bajerová, ${ }^{9}$ Rosan Meyer, ${ }^{10}$ Silvia Salvatore, ${ }^{11}$ Raanan Shamir, ${ }^{12}$ Anette Järvi, ${ }^{13}$ Ralf G Heine ${ }^{(D)},{ }^{13}$ MOSAIC Study Investigator Group

To cite: Vandenplas Y, Zhao Z-Y, Mukherjee R, et al. Assessment of the Cow's Milk-related Symptom Score (CoMiSS) as a diagnostic tool for cow's milk protein allergy: a prospective, multicentre study in China (MOSAIC study). BMJ Open 2022;12:e056641. doi:10.1136/ bmjopen-2021-056641

- Prepublication history and additional supplemental material for this paper are available online. To view these files, please visit the journal online (http://dx.doi.org/10.1136/ bmjopen-2021-056641).

Received 23 August 2021 Accepted 22 November 2021

Check for updates

(c) Author(s) (or their employer(s)) 2022. Re-use permitted under CC BY-NC. No commercial re-use. See rights and permissions. Published by BMJ.

For numbered affiliations see end of article.

Correspondence to Professor Zheng-Yan Zhao; zhaozy@zju.edu.cn

\section{ABSTRACT}

Objectives The MOSAIC study aimed to evaluate if the Cow's Milk-related Symptom Score (CoMiSS) can be used as a stand-alone diagnostic tool for cow's milk protein allergy (CMPA).

Design Single-blinded, prospective, multicentre diagnostic accuracy study.

Setting 10 paediatric centres in China.

Participants 300 non-breastfed infants (median age 16.1 weeks) with suspected CMPA.

Interventions After performing the baseline CoMiSS, infants commenced a cow's milk protein elimination diet with amino acid-based formula for 14 days. CoMiSS was repeated at the end of the elimination trial. Infants then underwent an open oral food challenge (OFC) with cow's milk-based formula (CMF) in hospital. Infants who did not react during the OFC also completed a 14-day home challenge with CMF. A diagnosis of CMPA was made if acute or delayed reactions were reported.

Primary outcome measures A logistic regression model for CoMiSS to predict CMPA was fitted and a receiveroperator characteristic (ROC) curve generated. An area under the curve (AUC) of $\geq 0.75$ was deemed adequate to validate CoMiSS as a diagnostic tool (target sensitivity $80 \%-90 \%$ and specificity $60 \%-70 \%$ ).

Results Of 254 infants who commenced the OFC, 250 completed both challenges, and a diagnosis of CMPA made in 217 (85.4\%). The median baseline CoMiSS in this group fell from 8 (IQR 5-10) to 5 (IQR 3-7) at visit 2 $(p<0.000000001)$, with a median change of -3 (IQR -6 to -1$)$. A baseline CoMiSS of $\geq 12$ had a low sensitivity (20.3\%), but high specificity $(87.9 \%)$ and high positive predictive value (91.7\%) for CMPA. The ROC analysis with an AUC of 0.67 fell short of the predefined primary endpoint.

Conclusions The present study did not support the use of CoMiSS as a stand-alone diagnostic tool for CMPA. Nevertheless, CoMiSS remains a clinically useful awareness tool to help identify infants with cow's milkrelated symptoms.
Strengths and limitations of this study

- The Cow's Milk-related Symptoms Score (CoMiSS), a widely used clinical awareness tool, was designed to help primary healthcare providers in identifying infants with cow's milk-related symptoms.

- This single-blinded, prospective, multicentre study (MOSAIC study) in 300 Chinese infants with suspected cow's milk allergy was adequately powered to assess if CoMiSS could be used as a stand-alone diagnostic tool for cow's milk protein allergy (CMPA).

- Following the Standards for Reporting of Diagnostic Accuracy Studies 2015 guidelines for reporting diagnostic accuracy studies, the study validated CoMiss as the index test against the reference standard for a diagnosis of CMPA, the oral food challenge (OFC).

- The primary study endpoint was based on the area under the curve of the receiver operating characteristic curve, in line with established diagnostic standards.

While the open OFC with predefined, objective stopping criteria is generally considered adequate for a diagnosis of CMPA in infants, the lack of doubleblinding of the OFC in the present study may have introduced confirmation bias into the challenge readout.

Trial registration number NCT03004729; Pre-results.

\section{INTRODUCTION}

Cow's milk protein allergy (CMPA) is one of the most common food allergies in infants and young children. ${ }^{1}$ The reported prevalence of CMPA in Europe, USA and China ranges from $0.5 \%$ up to $4.9 \% .^{2-5}$ While the immediate symptoms of immunoglobulin $\mathrm{E}$ (IgE)-mediated CMPA are readily recognised, 
the diagnosis of non-IgE mediated CMPA may be difficult due to the delayed onset of symptoms and overlap with other common paediatric conditions, such as infantile colic, gastro-oesophageal reflux, gastrointestinal infection or lactose intolerance ${ }^{6-9}$ In addition, apart from an oral food challenge (OFC) with cow's milk, there is currently no diagnostic test for non-IgE-mediated CMPA. ${ }^{10-12}$

The Cow's Milk-related Symptom Score (CoMiSS) was proposed by an expert panel in 2015 as an awareness tool for evaluating cow's milk-related symptoms in infants. ${ }^{13}$ The CoMiSS tool generates a score based on gastrointestinal, dermatological, respiratory and general symptoms (total score range 0-33) which may be associated with CMPA (online supplemental figure S1). A pilot study suggested that a CoMiSS $\geq 12$, in conjunction with a $\geq 50 \%$ score reduction following a cow's milk protein (CMP) elimination diet was suggestive of a diagnosis of CMPA. ${ }^{14} 15$ Several studies have assessed the diagnostic accuracy of CoMiSS in predicting CMPA, as well as tracking the clinical response to an elimination diet. ${ }^{15-19}$ While the clinical usefulness of predicting CMPA by CoMiSS was suggested in some of these studies, none was adequately designed to formally validate CoMiSS as a diagnostic tool. Two recent systematic reviews have concluded that CoMiSS may be useful in monitoring the clinical response to an elimination diet but found published data insufficient to recommend CoMiSS as a diagnostic tool for CMPA. ${ }^{12} 20$

The aim of the present study was to evaluate if CoMiSS, originally developed as an awareness tool, can be used as a stand-alone diagnostic tool for CMPA in infants. Secondary objectives included the assessment of sensitivity and specificity of the baseline CoMiSS before any dietary intervention, as well as the performance of individual clinical domains of CoMiSS. The primary endpoint of the present study was based on the area under the curve (AUC) of the receiver operating characteristic (ROC) for a logistic regression model used to predict CMPA. The ROC curve plots sensitivity against 1 -specificity for different thresholds of the probability of CMPA predicted by the logistic regression model. An AUC of 1.0 demonstrates perfect discrimination between diagnostic groups, and an AUC of 0.5 indicates discrimination no better than chance. There is debate about the usefulness of diagnostic tests with AUC values below 1.0. A common view is that an AUC of 0.8-0.9 generally indicates good to excellent diagnostic accuracy, while an AUC between 0.7 and 0.8 is at the lower range of adequate performance. ${ }^{21}$ Based on these considerations, the primary hypothesis was that CoMiSS had a high sensitivity (target range 80\%-90\%) and at least moderate specificity (target range 60\%-70\%) for predicting a diagnosis of CMPA, corresponding with an AUC of at least 0.75 on the ROC analysis.

\section{METHODS}

The MOSAIC study was conducted as a single-blinded, prospective, multicentre diagnostic accuracy study in 10 clinical sites in China between December 2016 and July 2018. All parents or legal guardians of participating infants provided written informed consent at the time of enrolment in the study. The overall conduct of the study was managed and monitored by a contract research organization, George Clinical, Sydney, Australia. The independent statistical analysis was performed by Cytel, USA. The MOSAIC study was prospectively registered (NCT03004729) prior to the enrolment of the first patient and the study protocol published in a peerreviewed journal before completion of the study. ${ }^{22}$

\section{Study design}

The assessment of CoMiSS as a diagnostic tool was conducted according to the Standards for Reporting of Diagnostic Accuracy Studies (STARD) 2015 guidelines for the reporting of diagnostic accuracy studies. ${ }^{23} \mathrm{CoMiSS}$ was defined as the index test and validated against the OFC as the reference standard, with a binary diagnostic CMPA status as 'absent'/ 'present'. Applying a group sequential design, the study population was randomised 1:1 into a 'training set' and a 'test set'. A logistic regression model based on the baseline CoMiSS and change from baseline to visit 2 was fitted to the training set, with CMPA status as the binary response. This model was then used to predict a diagnosis of CMPA in the test set and to generate the final ROC curve. The primary study endpoint to validate CoMiSS as a diagnostic tool was based on an AUC $\geq 0.75$ of the ROC curve in the test set.

\section{Interventions and outcome measures}

Non-breastfed infants under 6 months of age with any suspected cow's milk-related symptoms were consecutively enrolled according to the published inclusion criteria. ${ }^{22}$ Symptoms had to be present for at least 1 week and have developed within the first 2 months of commencing an infant formula containing intact CMP. After the baseline assessment which included the baseline CoMiSS (visit 1), infants were fed an amino acid-based formula (AAF; Alfamino, Nestlé Health Science, Switzerland) for 14 days. In infants 4 months of age or older, a CMP-free complementary diet was permitted. At the end of the elimination trial, CoMiSS was repeated (visit 2). In addition, the likelihood of CMPA was rated by investigators on a visual analogue scale (VAS; range $0=$ 'no CMPA' to 10 = 'definite CMPA') before and after the elimination trial.

After having completed the elimination trial, infants underwent an open OFC with a cow's milk-based infant formula (CMF; NAN1, Nestlé, Switzerland) over 6 hours in hospital, following the published PRACTical ALLergy (PRACTALL) consensus report. ${ }^{24}$ A detailed description of the OFC procedure is provided in the published protocol. ${ }^{22}$ Infants who attempted the OFC comprised the intent-to-treat (ITT) analysis population. The OFC was assessed based on predefined objective stopping criteria. ${ }^{24}$ Investigators performing the OFC were single-blinded to the CoMiSS result and response to AAF. Infants who did not react during the day 1 OFC in hospital were asked to continue with a 14-day, open CMF challenge at home. 
Subjects who completed both OFC phases were included in the per protocol (PP) analysis cohort. Any suspected allergic reactions (ie, vomiting, diarrhoea, recurrence of rectal bleeding, increased crying or regurgitation, eczema flare) during the home challenge were verified by the research staff during a clinical visit. A diagnosis of CMPA was made if infants had reacted either during the OFC in hospital or at home.

\section{Sample size calculation}

A pooled data analysis from three published trials using CoMiSS for the evaluation of hypoallergenicity of formulas intended for CMPA suggested that infants having a low CoMiSS (median, 5) after 1 month of a CMP elimination diet had a high likelihood of suffering from CMPA (OR, 0.83; 95\% CI 0.75 to 0.93; $\mathrm{p}=0.002$ ).${ }^{15}$ Based on that estimate, around 80 subjects with a positive OFC and 35 subjects with a negative $\mathrm{OFC}$ were required to test the study hypothesis regarding the AUC (with $90 \%$ power and at 5\% level of significance). For the group sequential design, two cohorts of 115 infants each (training set and test set) with and without CMPA were required (total of 230 infants). Assuming a non-completer rate of $25 \%-30 \%, 300$ infants needed to be enrolled.

\section{Interim analysis}

In view of uncertainties in the sample size estimation based on the expected prevalence of CMPA in the study cohort, a two-stage, adaptive, group sequential design was used. ${ }^{25}$ The main purpose of the interim analysis was to stop the trial early for efficacy or futility, or to increase the sample size if the interim AUC results for the ROC analysis fell in the 'promising zone'. ${ }^{26}$ The interim analysis was planned after 146 subjects had completed the OFC sequence and was carried out in an unblinded fashion by an independent Statistical Centre. The interim decision was made by an independent Data Monitoring Committee (iDMC).

\section{Statistical analysis}

Descriptive statistics (mean, median, SD) were used to summarise baseline characteristics of the study cohort, including CoMiSS. The IQR indicates the range between the 25th and 75th percentiles. The change in CoMiSS from baseline to visit 2 was assessed by the Wilcoxon signed-rank test for paired data, and the change in VAS scores by paired t-test. Based on $2 \times 2$ contingency tables of a CoMiSS cut-off $\geq 12$ by CMPA status, sensitivity, specificity, positive predictive values (PPVs) and negative predictive value (NPV) were calculated. This analysis was performed both for the baseline CoMiSS $\geq 12$ alone, and for CoMiSS $\geq 12$ in combination with a $50 \%$ percentage reduction from baseline. In addition, a sensitivity analysis assessed the diagnostic accuracy of CoMiSS for lower cut-off values ( $\geq 5$ to $\geq 11$ ). All statistical analyses were performed at the two-sided $5 \%$ level.

\section{Patient and public involvement}

There was no patient or public involvement.

\section{RESULTS}

Of 300 infants enrolled, one subject was excluded due to a missing baseline CoMiSS. The baseline characteristics of all infants with an evaluable CoMiSS $(n=299)$ are shown in table 1. Forty-six (15.3\%) infants were withdrawn by their parents before the OFC; the reasons for early withdrawal were not systematically documented. The remaining 254

Table 1 Baseline characteristics

\begin{tabular}{|c|c|c|c|c|c|}
\hline & & All infants & CMPA & Non-CMPA & Excluded \\
\hline & & $\mathrm{n}=\mathbf{2 9 9}$ & $\mathrm{n}=\mathbf{2 1 7}$ & $\mathrm{n}=33$ & $n=49$ \\
\hline \multirow[t]{2}{*}{ Gender, $n(\%)$} & Male & $178(59.5 \%)$ & $126(58.1 \%)$ & $21(63.6 \%)$ & $31(63.3 \%)$ \\
\hline & Female & $121(40.5 \%)$ & $91(41.9 \%)$ & $12(36.4 \%)$ & $18(36.7 \%)$ \\
\hline Gestational age, median (IQR) & Weeks & $39(38-40)$ & $39(38-40)$ & $39(38-40)$ & $39(38-40)$ \\
\hline Age at enrolment, median (IQR) & Weeks & $16.1(9.9-20.8)$ & $16.1(10.1-20.3)$ & $16.1(10.1-20.3)$ & $16.3(9.3-21.3)$ \\
\hline Race, $n(\%)$ & Asian & 299 (100\%) & 217 (100\%) & $33(100 \%)$ & $49(100 \%)$ \\
\hline \multirow[t]{2}{*}{ Method of delivery, $n(\%)$} & Vaginal & $151(50.7 \%)$ & $114(52.8 \%)$ & $17(51.5 \%)$ & $20(40.8 \%)$ \\
\hline & C-section & $147(49.3 \%)$ & $102(47.2 \%)$ & $16(48.5 \%)$ & $29(59.2 \%)$ \\
\hline Birth weight, median (IQR) & $\mathrm{kg}$ & $3.3(3.0-3.6)$ & $3.4(3.0-3.7)$ & $3.3(3.1-3.5)$ & $3.3(3.0-3.6)$ \\
\hline Birth length, median (IQR) & $\mathrm{cm}$ & $50.0(49.0-50.0)$ & $50.0(50.0-51.0)$ & $50.0(49.0-50.0)$ & $50.0(48.8-50.2$ \\
\hline Weight, median (IQR) & $\mathrm{kg}$ & $6.40(5.35-7.45)$ & $6.50(5.40-7.50)$ & $6.30(5.00-7.10)$ & $6.20(5.40-7.50)$ \\
\hline Length, median (IQR) & $\mathrm{cm}$ & $63.0(59.3-65.8)$ & $63.3(60.0-65.8)$ & $60.0(57.1-64.0)$ & $62.8(58.5-66.0)$ \\
\hline Head circumference, median (IQR) & $\mathrm{cm}$ & $40.0(38.5-42.0)$ & $40.0(38.5-42.0)$ & $39.5(38.5-42.4)$ & $40.0(39.0-42.0)$ \\
\hline Baseline CoMiSS, median (IQR) & & $8(5-10)$ & $8(6-11)$ & $5(4-10)$ & $7(5-8)$ \\
\hline
\end{tabular}

CMPA, cow's milk protein allergy; CoMiSS, Cow's Milk-related Symptom Score. 
$(84.6 \%)$ infants completed the CMP elimination diet with $\mathrm{AAF}$ and attempted the OFC (ITT cohort). Of the 254 infants in the ITT set, 250 infants completed both stages of the OFC (PP cohort). The parents of four infants did not want to proceed with the 14-day home challenge and withdrew from the study.

\section{Interim analysis}

The single interim look was carried out at $53.3 \%$ information fraction $(\mathrm{n}=160$ subjects), with an associated $0.35 \%$ alpha spent. The interim ROC analysis of the training set $(\mathrm{n}=80)$ found an AUC of 0.604 (sensitivity $69 \%$, specificity $51 \%$ ) which crossed the futility boundary. However, as recruitment at the time of the iDMC meeting had progressed fast and was nearly at the planned study end, the recommendation was to continue the trial to the final sample size of 300 infants. In addition, the statistical analysis plan was simplified towards a single final analysis of the entire ITT cohort.

\section{OFC outcomes}

Of 254 infants who attempted the OFC in hospital, 184 (72.4\%) had a clinical reaction to CMF during the day 1 hospital challenge. Of the remaining $70(27.6 \%)$ infants, $66(26.0 \%)$ completed the 14-day home challenge. Of these, 33 (13.0\%) were diagnosed with CMPA. Combining positive challenges from both OFC stages, a total of 217 (85.4\%) infants had CMPA. A diagnosis of CMPA was excluded in $33(13.0 \%)$ infants. A detailed description of the OFC outcomes has previously been published. ${ }^{27}$

Atopic dermatitis was by far the most common presentation in both groups ( $54 \%$ vs $45 \%)$. There were minor differences in the clinical characteristics between the CMPA and non-CMPA groups. While rectal bleeding and regurgitation/vomiting were more commonly seen in infants with CMPA, infants in the non-CMPA group had a greater representation of constipation, persistent crying and respiratory symptoms. However, none of these differences was statistically significant, in part due to low numbers. The clinical presentation of infants in the PP cohort is summarised in table 2.

\section{CoMiSS scores at baseline and change after the elimination trial}

In the population of infants with evaluable data, the median baseline CoMiSS was 8 (IQR 5-10; range 0-24). Infants with CMPA had a higher CoMiSS at baseline, compared with CMPA-negative infants; 8 (IQR 6-11) versus 5 (IQR 4-10). In the infants with confirmed CMPA, there was a highly significant reduction in CoMiSS from 8 (IQR 6-11) to 5 (IQR 3-7) from baseline to visit $2(\mathrm{p}<0.000000001)$, with a median reduction by -3 (IQR -6 to -1$)$. A lesser reduction was observed in the infants without CMPA where CoMiSS fell from 5 (IQR 4-10) to 3.5 (IQR 2-7), with a median change of $-2(\mathrm{IQR}-5$ to -1$) ; \mathrm{p}=0.0013$. The absolute decrease in CoMiSS values from baseline to visit 2 was significantly greater in infants with high baseline scores (adjusted $\left.\mathrm{r}^{2}=0.568 ; \mathrm{p}<0.0000001\right)$. CoMiSS scores at baseline and visit 2 are summarised in figure 1 and online supplemental table S1 (in supplemental materials).

\section{Individual CoMisS domains}

In the present study, the composite CoMiSS was mainly made up of scores from the stool, eczema and respiratory symptom domains, while crying and regurgitation contributed to a much lesser extent. In the CMPA group, the reduction in scores for each clinical domain following the elimination diet was highly significant, while in the non-CMPA group changes were less pronounced or non-significant (crying, regurgitation, urticaria). Thirty-nine $(15.6 \%)$ infants in the PP cohort had presented with rectal bleeding, suggestive of food protein-induced proctocolitis. As rectal bleeding is not part of the CoMiSS design, this clinical manifestation did not contribute to composite scores. Depending on concomitant symptoms, infants with rectal bleeding had total CoMiSS values ranging from 0 to 3 . The skin scores

\begin{tabular}{|c|c|c|c|}
\hline Main clinical presentation & CMPA $n=217$ & No CMPA $n=33$ & Total $n=250$ \\
\hline Atopic dermatitis/eczema & $118(54.4 \%)$ & $15(45.5 \%)$ & $133(53.2 \%)$ \\
\hline Rectal bleeding & $35(16.1 \%)$ & $4(12.1 \%)$ & 39 (15.6\%) \\
\hline Persistent diarrhoea & $23(10.6 \%)$ & $4(12.1 \%)$ & $27(10.8 \%)$ \\
\hline Regurgitation/vomiting & $23(10.6 \%)$ & $1(3.0 \%)$ & 24 (9.6\%) \\
\hline Constipation & $6(2.8 \%)$ & $3(9.1 \%)$ & $9(3.6 \%)$ \\
\hline Persistent crying/irritability & $5(2.3 \%)$ & $2(6.1 \%)$ & $7(2.8 \%)$ \\
\hline Poor weight gain & $5(2.3 \%)$ & $0(0 \%)$ & $5(2.0 \%)$ \\
\hline Respiratory symptoms & $0(0 \%)$ & $3(9.1 \%)$ & $3(1.2 \%)$ \\
\hline Feeding difficulties & $0(0 \%)$ & $1(3.0 \%)$ & $1(0.4 \%)$ \\
\hline Other/not documented & $2(0.9 \%)$ & $0(0 \%)$ & $2(0.8 \%)$ \\
\hline
\end{tabular}

CMPA, cow's milk protein allergy. 

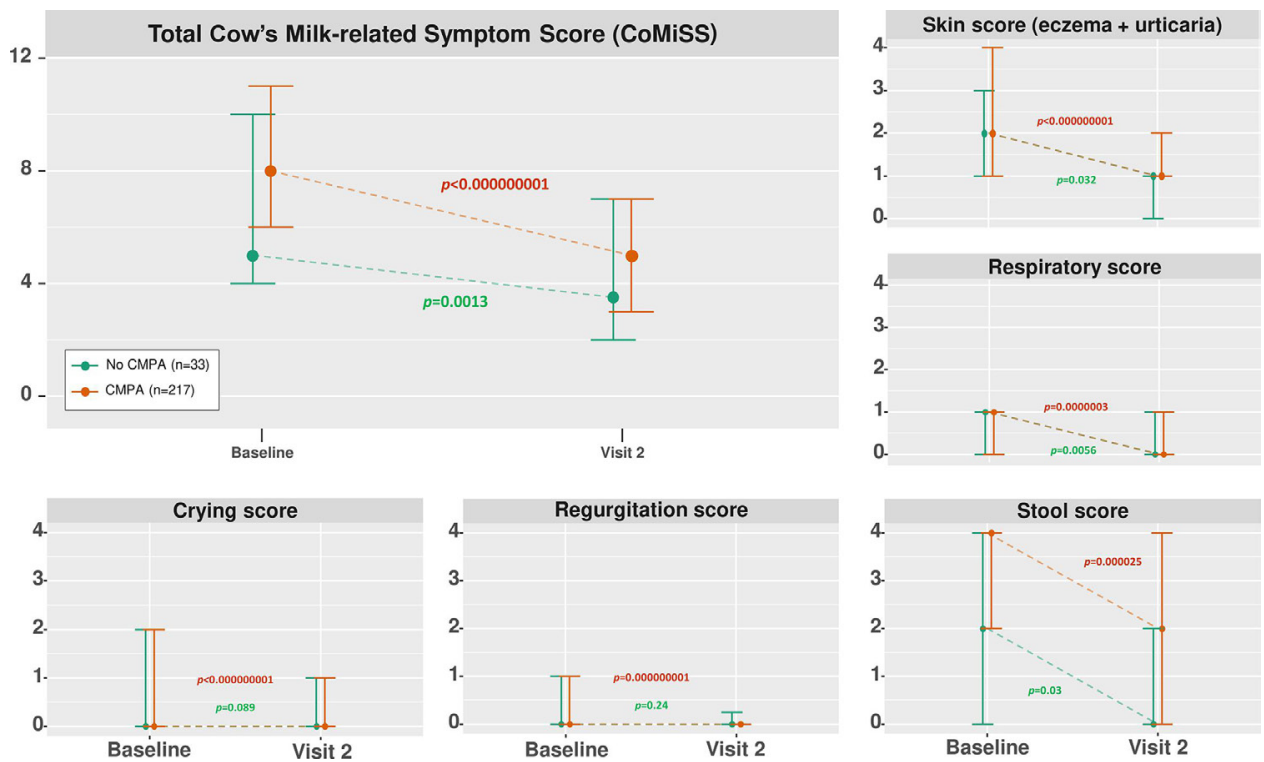

Figure 1 The change in Cow's Milk-related Symptom Score (CoMiSS) from baseline to the end of the elimination diet trial (visit 2) is shown for the 217 infants with confirmed cow's milk protein allergy (CMPA) and 33 infants without CMPA (median, IQR). The change in median CoMiSS is depicted for the total composite score, as well as for each of its five clinical domains (crying, regurgitation, stool, skin and respiratory scores).

were almost entirely due to atopic dermatitis, as only 11 infants had urticaria at baseline (CMPA $n=9$, non-CMPA $\mathrm{n}=2$ ). Urticaria persisted to visit 2 in 4 infants (CMPA $\mathrm{n}=3$, non-CMPA $\mathrm{n}=1$ ). The change in CoMiSS for individual clinical domains is summarised in figure 1 and online supplemental table S1.

\section{Diagnostic accuracy assessment of CoMiSS}

The study flow diagram according to the 'STARD' is shown in figure $2 .{ }^{23}$ Sensitivity, specificity, PPV and NPV were calculated based on $2 \times 2$ contingency tables for the PP cohort (online supplemental table S2). Applying a cut-off of $\geq 12$, the baseline CoMiSS had a low sensitivity of $20.3 \%$, but was highly specific for CMPA $(87.9 \%)$ and had a high PPV $(91.7 \%)$. A sensitivity analysis of a range for cut-off values showed that for lower cut-off values the

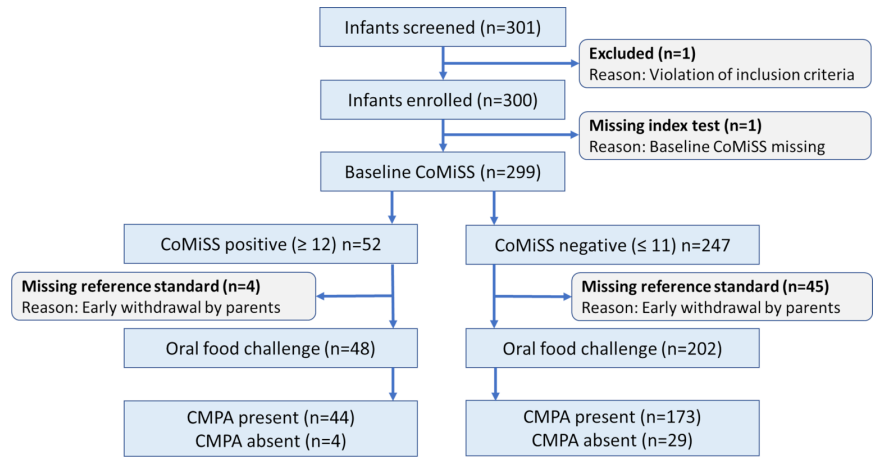

Figure 2 The flow diagram summarises the disposition of subjects according to the Standards for Reporting of Diagnostic Accuracy Studies 2015 guidelines for reporting diagnostic accuracy studies. The index test (baseline Cow's Milk-related Symptom Score (CoMiSS)) was validated against the oral food challenge as the reference standard for diagnosing cow's milk protein allergy (CMPA). sensitivity generally improved, but at the expense of specificity. Combining the baseline cut-off with a $\geq 50 \%$ reduction in CoMiSS also improved the specificity but further reduced the sensitivity. The best diagnostic accuracy was found for a baseline CoMiSS $\geq 6$ (sensitivity $78.8 \%$, specificity $51.5 \%$ ); online supplemental table S2.

Similar findings were observed in the single final ROC analysis of the PP cohort. The estimated logistic regression model was $\left(\right.$ logit ${ }_{\mathrm{CMPA}}=0.378+(0.252 \times$ baseline CoMiSS $)+(0.065 \times$ delta CoMiSS V1-V2 $)-(0.017 \times$ interaction of baseline and delta CoMiSS V1-V2)). Based on this ROC model, the AUC was 0.67 which fell short of the primary study endpoint of $A U C \geq 0.75$; figure 3 .

\section{Investigator assessment of likelihood of CMPA by VAS}

Paired VAS data on investigator assessment of the likelihood of CMPA were available for 251 (99\%) infants in the ITT cohort. The mean VAS slightly decreased from 7.15 (SD 1.47) at baseline to 6.76 (SD 2.1) at visit 2; paired t-test $\mathrm{p}=0.0164$. In infants with CMPA $(\mathrm{n}=217)$, investigators were less confident of a diagnosis of CMPA with a decrease in VAS from 7.26 (SD 1.43) to 6.86 (SD 2.07); paired t-test $\mathrm{p}=0.0039$. Overall, there was poor correlation between the VAS at baseline or visit 2 with corresponding CoMiSS values (baseline, $\mathrm{r}=0.1$; visit $2, \mathrm{r}=0.16$ ).

\section{Impact of birth mode on baseline CoMiSS and CMPA}

In the ITT set, 131 infants were born via caesarean section, and 118 via vaginal delivery. There was no significant association between birth mode and CMPA status (CMPA rate: caesarean section 94\% $(114 / 131)$ vs vaginal delivery $86 \%$ (102/118); $\left.\chi^{2}=0.018 ; \mathrm{p}=0.89\right)$. In addition, there were no statistically significant differences between the baseline CoMiSS of infants born via caesarean section, 


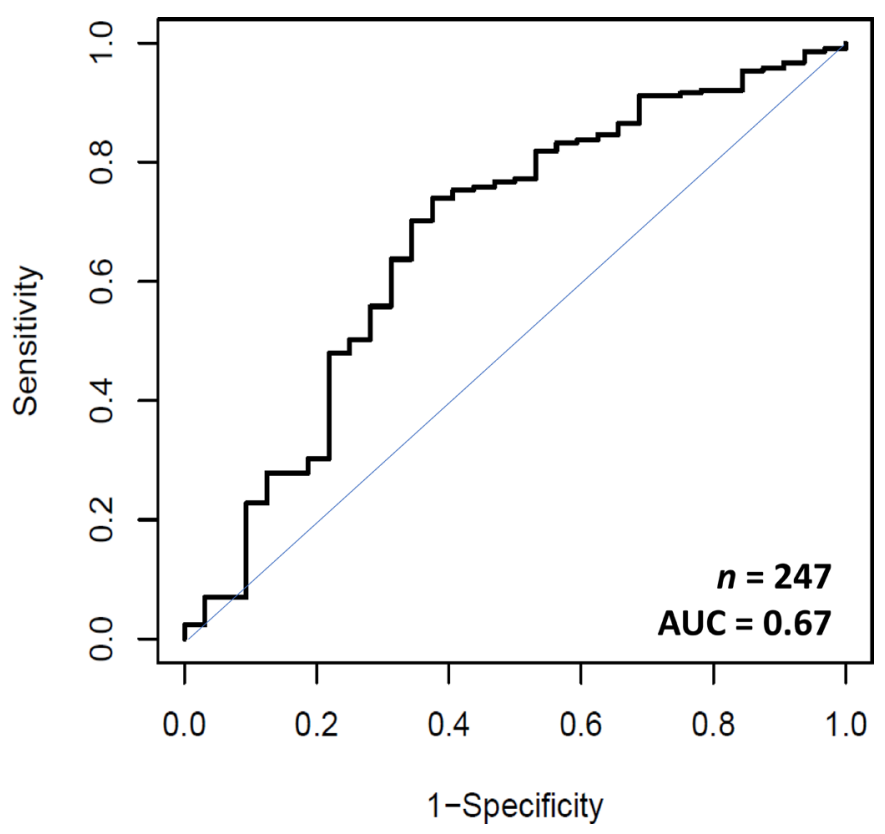

Figure 3 The receiver operating characteristic) curve is based on the logistic regression model of the Cow's Milkrelated Symptom Score (CoMiSS) and plots the sensitivity against (1-specificity) of CoMiSS for predicting cow's milk protein allergy status. The area under the curve (AUC) of 0.67 remained below the predefined primary study endpoint of $\geq 0.75$.

compared with vaginally delivered infants (median baseline CoMiSS: 7 (IQR 6-10) vs 8 (IQR 5-10.5); N.S.).

\section{Reporting of other secondary study objectives}

Of the 11 secondary objectives defined in the published protocol, ${ }^{22} 7$ are reported here. The anthropometric outcomes to 9 months of age of the CMPA cohort have previously been published. ${ }^{27}$ The assessment of the impact of a family history of atopy on baseline CoMiSS and the prevalence of CMPA was omitted due to missing data. We also omitted the assessment of compliance with the elimination diet and of the therapeutic effect of an AAF-based diet as supporting data were not collected.

\section{DISCUSSION}

The present diagnostic accuracy study conducted in 300 Chinese infants was the first to attempt a formal validation of CoMiSS as a stand-alone diagnostic tool for CMPA. CoMiSS was originally established as a clinical awareness tool for cow's milk-related symptoms in infancy. Currently, a definitive CMPA diagnosis requires a formal CMP elimination and OFC sequence, particularly in infants with suspected nonIgE-mediated CMPA presenting with a range of non-specific gastrointestinal or behavioural symptoms. ${ }^{10} 12$ However, in clinical practice the confirmatory challenge is often omitted or delayed. If validated as a diagnostic tool, CoMiSS could potentially simplify the complex diagnostic process and reduce the need for confirmatory OFCs.

The present study missed its primary endpoint and did not support the use of CoMiSS as a stand-alone diagnostic tool for CMPA. More precisely, the AUC of 0.67 remained below the predefined cut-off of at least 0.75 . CoMiSS fell significantly from baseline to the end of the CMP elimination diet, with a greater reduction seen in the infants with CMPA. CoMiSS may therefore have a role in monitoring the response to a diagnostic elimination diet, as previously suggested. ${ }^{15}$ The significant score reduction observed in the non-CMPA group may be due to several factors, including the treatment of atopic dermatitis with moisturisers and/or topical corticosteroids which is likely to have reduced skin findings and which may indirectly have improved crying or sleep behaviours. ${ }^{28}$ In addition, a non-specific placebo effect after formula change, parental support during a clinical trial and a time effect in conditions that tend to improve spontaneously (eg, viral gastrointestinal or respiratory infections) may also have contributed to a decrease in scores.

Applying the recommended cut-off of $\geq 12$, with or without a 50\% score reduction after the elimination trial, CoMiSS was highly specific (range $87.5 \%-87.9 \%$ ) for CMPA but lacked sensitivity (range 14.0\%-20.3\%); online supplemental table S2. Lowering the cut-off values generally improved the sensitivity, but at the expense of specificity, with the best combination achieved for a CoMiSS cut-off of $\geq 6$. This aligns with another recent Chinese study which found the bestperforming diagnostic cut-off values between 5 and $6 .{ }^{19}$ By comparison, the optimum diagnostic cut-off in a recent Italian study ${ }^{17}$ was $\geq 9$ which corresponds with the 95th percentile in a study on CoMiSS in 413 presumed healthy European infants from Belgium, Italy, Poland and Spain. ${ }^{29}$ In that study, the median CoMiSS was 3 which is similar to the value of 3.5 (IQR 2-7) found in the non-CMPA at visit 2. By contrast, infants with CMPA in the present study had higher CoMiSS values at visit 2 (median 5; IQR 3-7), suggesting differences between populations or an incomplete response after 2 weeks of AAF treatment.

The composite CoMiSS in Chinese infants in our study was mainly made up of scores for the skin, respiratory and stool domains. This included more than $50 \%$ of infants with atopic dermatitis, and about 15\% had presented with rectal bleeding. Even though atopic dermatitis and rectal bleeding are well-recognised manifestation of CMPA, ${ }^{1} 1028$ the high proportion of infants in the present study with these conditions differs from the clinical spectrum described in European studies. ${ }^{17}$ The design of CoMiSS emphasises the importance of persistent crying, regurgitation and abnormal stools, in line with clinical practices in Europe ${ }^{13}$ Accordingly, two studies from Italy and India mostly included infants with a combination of these symptoms. ${ }^{16}{ }^{17}$ By contrast, a study on CoMiSS from Turkey found that the presence of eczema was the most useful predictor of CMPA. ${ }^{18} \mathrm{~A}$ low number of symptoms, low scores for crying and regurgitation, as well as a significant number of infants with rectal bleeding may explain the generally lower CoMiSS values (median, 8 ) in the present study, compared with previous trials. ${ }^{1719}$ These data suggest regional differences in the clinical spectrum of CMPA, as well as potential differences in perceptions by parents and primary care practitioners, for example, regarding persistent crying and regurgitation in infants. Differences in language 
and interpretation of CoMiSS may also affect its performance as a diagnostic tool. ${ }^{19}{ }^{29}$ Future adaptations of the CoMiSS tool should therefore re-evaluate lower cut-off values in an attempt to improve the ability to identify infants with CMPA.

The present study had several limitations. The ITT cohort consisted to $85 \%$ of infants with CMPA. The early withdrawal of $46(15 \%)$ enrolled participants due to possible nonresponse to the elimination diet may have enriched the ITT cohort for a diagnosis of CMPA. In addition, the the lack of blinding during the OFC may have have led to an overestimate of CMPA due to confirmation bias. However, singleblinded, open OFC with predefined, objective stopping criteria are generally considered sufficient for a diagnosis of CMPA in infants, as even double-blind, placebo-controlled food challenges in this age group may be prone to errors. ${ }^{30} 31$ A significant proportion of infants in our study had residual atopic dermatitis at the time of the OFC which could point to concomitant food allergies (eg, egg allergy) or incomplete treatment. Skin flares unrelated to CMPA may thus have confounded challenge outcomes. In addition, rectal bleeding which was not captured by CoMiSS may have reduced the diagnostic accuracy of the tool. The present study did not define the type of CMPA (ie, IgE-mediated vs non-IgE-mediated). While the clinical presentation of infants was mostly suggestive of non-IgE-mediated CMPA, there was an unexpectedly high rate of immediate and possibly IgEmediated reactions during the day 1 OFC. By contrast, there were only a small number of infants with urticarial reactions as a clinical marker of IgE-mediated CMPA. As skin prick testing or serum IgE antibody testing was not included in the protocol, this discrepancy could not be resolved. We were therefore unable to assess if CoMiSS performs equally well in infants with IgE-mediated or non-IgE-mediated CMPA. Similarly, our study only assessed CoMiSS in formula-fed infants, and its usefulness in breastfed infants requires further study.

In summary, the present study in Chinese infants found that CoMiSS lacked accuracy as a stand-alone diagnostic test for CMPA. The spectrum of CMPA manifestations was associated with generally low CoMiSS values which overlapped with the scores found in presumably healthy European infants. ${ }^{29}$ These findings indicate that the clinical spectrum of CMPA may differ between regions, with a lower prevalence of infantile colic and functional gastrointestinal symptoms in infants in China compared with European infants. ${ }^{32-34} \mathrm{In}$ the Chinese infants of this study, a baseline CoMiSS $\geq 12$ was highly predictive and specific for CMPA but lacked sensitivity. Other studies, as well as data in healthy infants, suggested that a lowering of the CoMiSS cut-off could improve its overall performance. ${ }^{1729}$ At this stage, a diagnosis of CMPA still requires both a clear response to CMP elimination and a clinical recurrence of symptoms during a confirmatory OFC. ${ }^{10}$ In this process, CoMiSS may be used to document the change in symptoms after a CMP elimination. ${ }^{12}{ }^{15}$ If a confirmatory OFC is not feasible or declined by parents, a baseline CoMiSS of 12 or higher, in conjunction with an unequivocal clinical response to CMP elimination, was highly specific and predictive and may thus support a diagnosis of CMPA. Of note, CoMiSS is not suitable for infants with suspected food protein-induced proctocolitis or food protein-induced enteropathy syndrome (FPIES) as these conditions are outside its scope and design. ${ }^{13}$ In conclusion, CoMiSS did not demonstrate the required accuracy of a stand-alone diagnostic test for CMPA but remains a clinically useful awareness tool in primary care for the early identification of infants with cow's milk-related symptoms. ${ }^{13} 14$

\section{Author affiliations}

${ }^{1}$ Vrije Universiteit Brussel, UZ Brussel, KidZ Health Castle, Brussels, Belgium ${ }^{2}$ Children's Hospital Zhejiang, University School of Medicine, Zhejiang University, Hangzhou, China

${ }^{3}$ Cytel Inc, Waltham, Massachusetts, USA

${ }^{4}$ Department of Paediatrics, Hôpital Necker-Enfants Malades, Paris, France ${ }^{5}$ Department of Infants and Adolescents, University Hospitals Geneva, Geneva, Switzerland

${ }^{6}$ New Children's Hospital, Helsinki University Central Hospital, Helsinki, Finland ${ }^{7}$ Paediatric Gastroenterology, La Fe University and Polytechnic Hospital, Valencia, Spain

${ }^{8}$ Department of Paediatrics, Medical University of Warsaw, Warszawa, Poland ${ }^{9}$ Research Institute, Department of Paediatrics, Marien-Hospital Wesel, Wesel, Germany

${ }^{10}$ Department of Paediatrics, Imperial College London, London, UK ${ }^{11}$ Department of Paediatrics, Hospital 'F. Del Ponte', University of Insubria, Varese, Italy

${ }^{12}$ Institute for Gastroenterology, Nutrition and Liver Disease, Schneider Children's Medical Center, Sackler Faculty of Medicine, Tel Aviv University, Tel Aviv, Israel

${ }^{13}$ Nestlé Health Science, Vevey, Switzerland

Acknowledgements The authors wish to thank all families and research staff involved with the study. The contribution of the principal investigators in China (MOSAIC Study Investigator Group) and research staff at each of the 10 study sites is gratefully acknowledged.

Collaborators MOSAIC Study Investigator Group: Zheng-Yan Zhao, Children's Hospital Zhejiang, University School of Medicine, Hangzhou, Zhejiang, China (lead); Xiaomei Tong, University Third Hospital, Beijing, China; Yan Hu, Children's Hospital of Chongqing Medical University, Chongqing, China; Liyan Zhang, Children's Hospital of Fuzhou, Fujian, China; Jieling Wu, Maternal and Child Health Care Hospital, Guangzhou, Guangdong, China; Shufen Yang, Second Affiliated Hospital of Harbin Medical University, Ha'erbin, Heilongjiang, China; Xiao Qin Li, Children's Hospital of Zhengzhou, Henan, China; Yan Zhong, Hunan Children's Hospital, Hunan, China; Jinjin Chen, Children's Hospital of Shanghai, Shanghai, China; Qing Zhao, Children's Hospital of Shanxi Women Health Center, Taiyuan, Shanxi, China.

Contributors YV and RaM were responsible for the concept and design of the study. Z-YZ was the lead principal investigator for China who coordinated the study execution in the 10 study sites. RaM performed the independent statistical analysis. All authors have actively participated in the study readout and interpretation of findings. YV and RGH wrote the first draft of the manuscript. All authors participated in the editing process and have approved the final version of the manuscript. YV and Z-YZ have contributed equally to the manuscript and are joint first authors. YV and $\mathrm{RGH}$ are responsible for the overall content as the guarantor.

Funding This trial was sponsored by Nestlé Health Science (Grant number: not applicable).

Competing interests YV has participated as a clinical investigator, and/or advisory board member, and/or consultant, and/or speaker for Abbott Nutrition, Biogaia, Biocodex, Danone, Hero, Hipp, Johnson \& Johnson, Mead Johnson, Merck, Menarini, Nestlé Nutrition Institute, Nutricia, Orafti, Pfizer, Phacobel, Sari Husada, Shire (Movetis), Sucampo, Takeda, United Pharmaceuticals, Wyeth and Yakult. CD has participated as a clinical investigator, and/or advisory board member, and/or consultant, and/or speaker for Abbott Nutrition, Danone, Novalac, Nestlé Nutrition Institute, Nutricia, Sodilac, United Pharmaceuticals and Wyeth. PE has received lecture honoraria from Danone and Sodilac, and has received research grants from Nestlé. CR-K has participated as consultant and/or speaker for Mead Johnson International, Hero Institute, Alter-Nutriben, Danone and Nestlé. HS has participated as a clinical investigator and/or speaker for Ausnutria, Arla, Biogaia, Biocodex, Danone, Dicofarm, Else Nutrition, HiPP, Nestlé, Nestlé Nutrition Institute, Nutricia, Mead Johnson, Myor and Merck. KB has received lecture honoraria from Nestlé. 
SS has participated as a clinical investigator/, and/or advisory board member, and/or consultant, and/or speaker for Danone, Dicofarm, DVA, Nestlé and United Pharmaceuticals. RS has participated as clinical investigator, and/or advisory board member, and/or consultant and/or speaker for Abbott, Else, Nestlé Nutrition Institute, NGS and Nutricia. AJ and RGH are full-time employees of Nestlé Health Science. The other authors have disclosed no conflict of interest relevant to this article.

Patient consent for publication Not applicable.

Ethics approval The present study was approved by the Medical Ethics Committee at Hunan Children's Hospital, Hunan, China (HCHLL-2016-016). Participants gave informed consent to participate in the study before taking part.

Provenance and peer review Not commissioned; externally peer reviewed.

Data availability statement Data are available upon reasonable request. Data are available upon request from the Chief Science \& Medical Officer, Nestlé Health Science, $\mathrm{CH}-1800$ Vevey, Switzerland.

Supplemental material This content has been supplied by the author(s). It has not been vetted by BMJ Publishing Group Limited (BMJ) and may not have been peer-reviewed. Any opinions or recommendations discussed are solely those of the author(s) and are not endorsed by BMJ. BMJ disclaims all liability and responsibility arising from any reliance placed on the content. Where the content includes any translated material, BMJ does not warrant the accuracy and reliability of the translations (including but not limited to local regulations, clinical guidelines, terminology, drug names and drug dosages), and is not responsible for any error and/or omissions arising from translation and adaptation or otherwise.

Open access This is an open access article distributed in accordance with the Creative Commons Attribution Non Commercial (CC BY-NC 4.0) license, which permits others to distribute, remix, adapt, build upon this work non-commercially, and license their derivative works on different terms, provided the original work is properly cited, appropriate credit is given, any changes made indicated, and the use is non-commercial. See: http://creativecommons.org/licenses/by-nc/4.0/.

Author note CoMiSSTM is a Registered Trademark of Sociéte des Produits Nestlé SA, Switzerland.

\section{ORCID iD}

Ralf G Heine http://orcid.org/0000-0002-3343-0379

\section{REFERENCES}

1 Heine RG, Elsayed S, Hosking CS, et al. Cow's milk allergy in infancy. Curr Opin Allergy Clin Immunol 2002;2:217-25.

2 Schoemaker AA, Sprikkelman AB, Grimshaw KE, et al. Incidence and natural history of challenge-proven cow's milk allergy in European children--EuroPrevall birth cohort. Allergy 2015;70:963-72.

3 Gupta RS, Springston EE, Warrier MR, et al. The prevalence, severity, and distribution of childhood food allergy in the United States. Pediatrics 2011;128:e9-17.

4 Yang M, Tan M, Wu J, et al. Prevalence, characteristics, and outcome of cow's milk protein allergy in Chinese infants: a population-based survey. JPEN J Parenter Enteral Nutr 2019;43:803-8.

5 Kvenshagen B, Halvorsen R, Jacobsen M. Adverse reactions to milk in infants. Acta Paediatr 2008;97:196-200.

6 Heine RG. Gastroesophageal reflux disease, colic and constipation in infants with food allergy. Curr Opin Allergy Clin Immunol 2006;6:220-5

7 Omari T, Tobin JM, McCall L, et al. Characterization of upper gastrointestinal motility in infants with persistent distress and NonIgE-mediated cow's milk protein allergy. J Pediatr Gastroenterol Nutr 2020;70:489-96.

8 Guarino A, Albano F, Ashkenazi S. European Society for Paediatric Gastroenterology, Hepatology, and Nutrition; European Society for Paediatric Infectious Diseases. European Society for Paediatric Gastroenterology, Hepatology, and Nutrition/European Society for Paediatric Infectious diseases evidence-based guidelines for the management of acute gastroenteritis in children in Europe. $J$ Pediatr Gastroenterol Nutr 2008;46:S81-122.

9 Heine RG, AlRefaee F, Bachina P, et al. Lactose intolerance and gastrointestinal cow's milk allergy in infants and children - common misconceptions revisited. World Allergy Organ J 2017;10:41.

10 Koletzko S, Niggemann B, Arato A, et al. Diagnostic approach and management of cow's-milk protein allergy in infants and children: ESPGHAN GI Committee practical guidelines. J Pediatr Gastroenterol Nutr 2012;55:221-9.

11 Venter C, Brown T, Meyer R, et al. Better recognition, diagnosis and management of non-IgE-mediated cow's milk allergy in infancy: iMAP-an international interpretation of the MAP (milk allergy in primary care) guideline. Clin Transl Allergy 2017;7:26.

12 Calvani M, Anania C, Cuomo B, et al. Non-lgE- or mixed IgE/NonIgE-Mediated gastrointestinal food allergies in the first years of life: old and new tools for diagnosis. Nutrients 2021;13. doi:10.3390/ nu13010226. [Epub ahead of print: 14 Jan 2021]

13 Vandenplas Y, Dupont C, Eigenmann P, et al. A workshop report on the development of the cow's milk-related symptom score awareness tool for young children. Acta Paediatr 2015;104:334-9.

14 Vandenplas Y, Steenhout P, Grathwohl D. A pilot study on the application of a symptom-based score for the diagnosis of cow's milk protein allergy. SAGE Open Med 2014;2:205031211452342.

15 Vandenplas $Y$, Steenhout $P$, Järvi $A$, et al. Pooled analysis of the cow's milk-related-symptom-score (CoMiSS ${ }^{\mathrm{TM}}$ ) as a predictor for cow's milk related symptoms. Pediatr Gastroenterol Hepatol Nutr 2017;20:22-6.

16 Prasad R, Venkata RSA, Ghokale P, et al. Cow's milk-related symptom score as a predictive tool for cow's milk allergy in Indian children aged 0-24 months. Asia Pac Allergy 2018;8:e36.

17 Salvatore S, Bertoni E, Bogni F, et al. Testing the cow's milk-related symptom score (CoMiSS ${ }^{\mathrm{TM}}$ ) for the response to a cow's milk-free diet in infants: a Prospective Study. Nutrients 2019;11:2402.

18 Selbuz SK, Altuntas C, Kansu A, et al. Assessment of cows milkrelated symptom scoring awareness tool in young Turkish children. $J$ Paediatr Child Health 2020;56:1799-805.

19 Zeng Y, Zhang J, Dong G, et al. Assessment of cow's milk-related symptom scores in early identification of cow's milk protein allergy in Chinese infants. BMC Pediatr 2019;19:191.

20 Thompson G, Zhelev Z, Peters J. Symptom scores in the diagnosis of pediatric cow's milk protein allergy: A systematic review. Pediatr Allergy Immunol 2021.

21 Mandrekar JN. Receiver operating characteristic curve in diagnostic test assessment. J Thorac Oncol 2010;5:1315-6.

22 Vandenplas Y, Mukherjee R, Dupont C, et al. Protocol for the validation of sensitivity and specificity of the cow's milk-related symptom score (CoMiSS) against open food challenge in a single-blinded, prospective, multicentre trial in infants. BMJ Open 2018;8:e019968.

23 Bossuyt PM, Cohen JF, Gatsonis CA. STARD 2015: updated reporting guidelines for all diagnostic accuracy studies. Ann Trans/ Med 2015;2016:85.

24 Sampson HA, Gerth van Wijk R, Bindslev-Jensen C, et al. Standardizing double-blind, placebo-controlled oral food challenges: American Academy of Allergy, Asthma \& Immunology-European Academy of Allergy and Clinical Immunology PRACTALL consensus report. J Allergy Clin Immunol 2012;130:1260-74.

25 Hwang IK, Shih WJ, De Cani JS. Group sequential designs using a family of type I error probability spending functions. Stat Med 1990:9:1439-45.

26 Mehta CR, Pocock SJ. Adaptive increase in sample size when interim results are promising: a practical guide with examples. Stat Med 2011;30:3267-84.

27 Vandenplas Y, Dupont C, Eigenmann P, et al. on behalf of the MOSAIC Study Investigator Group. Growth in infants with cow's milk protein allergy fed an amino acid-based formula. Pediatr Gastroenterol Hepatol Nutr 2021;24:392-402.

28 Giannetti A, Cipriani F, Indio V, et al. Influence of atopic dermatitis on cow's milk allergy in children. Medicina 2019;55:460.

29 Vandenplas Y, Salvatore S, Ribes-Koninckx C, et al. The cow milk symptom score (CoMiSSTM) in presumed healthy infants. PLoS One 2018;13:e0200603.

30 Koletzko S, Heine RG. Non-lgE mediated cow's milk allergy in EuroPrevall. Allergy 2015;70:1679-80.

31 Niggemann B, Beyer K. Pitfalls in double-blind, placebo-controlled oral food challenges. Allergy 2007;62:729-32.

32 Liu W, Xiao L-ping, Li Y, et al. [Epidemiology of mild gastrointestinal disorders among infants and young children in Shanghai area]. Zhonghua Er Ke Za Zhi 2009;47:917-21.

33 Huang Y, Tan SY, Parikh P, et al. Prevalence of functional gastrointestinal disorders in infants and young children in China. BMC Pediatr 2021;21:131.

34 Chew KS, Em JM, Koay ZL, et al. Low prevalence of infantile functional gastrointestinal disorders (FGIDs) in a multi-ethnic Asian population. Pediatr Neonatol 2021;62:49-54. 